\title{
Beneficial effects of TQRF and TQ nano- and conventional emulsions on memory deficit, lipid peroxidation, total antioxidant status, antioxidants genes expression and soluble Ab levels in high fat-cholesterol diet-induced rats
}

\begin{abstract}
The study determined the effect of thymoquinone rich fraction (TQRF) and thymoquinone (TQ) in the forms of nano- and conventional emulsions on learning and memory, lipid peroxidation, total antioxidant status, antioxidants genes expression and soluble $\beta$-amyloid $(\mathrm{A} \beta)$ levels in rats fed with a high fat-cholesterol diet (HFCD). The TQRF was extracted from Nigella sativa seeds using a supercritical fluid extraction system and prepared into nanoemulsion, which later named as TQRF nanoemulsion (TQRFNE). Meanwhile, TQ was acquired commercially and prepared into thymoquinone nanoemulsion (TQNE). The TQRF and TQ conventional emulsions (CE), named as TQRFCE and TQCE, respectively were studied for comparison. Statin (simvastatin) and non-statin (probucol) cholesterol-lowering agents, and a mild-to-severe Alzheimer's disease drug (donepezil) were served as control drugs. The Sprague Dawley rats were fed with HFCD for 6 months, and treated with the intervention groups via oral gavage daily for the last 3 months. As a result, HFCD-fed rats exhibited hypercholesterolaemia, accompanied by memory deficit, increment of lipid peroxidation and soluble $A \beta$ levels, decrement of total antioxidant status and down-regulation of antioxidants genes expression levels. TQRFNE demonstrated comparable effects to the other intervention groups and control drugs in serum biomarkers as well as in the learning and memory test. Somehow, TQRFNE was more prominent than those intervention groups and control drugs in brain biomarkers concomitant to gene and protein expression levels. Supplementation of TQRFNE into an HFCD thus could ameliorate memory deficit, lipid peroxidation and soluble $A \beta$ levels as well as improving the total antioxidant status and antioxidants genes expression levels.
\end{abstract}

Keyword: Hypercholesterolemia; Memory deficit; Nanoemulsion; Thymoquinone; Thymoquinone-rich fraction; $\beta$-amyloid 